\title{
BMJ Open Multicentre, prospective, randomised, controlled, blinded-endpoint study to evaluate the efficacy and safety of pterygopalatine ganglion pulsed radiofrequency treatment for cluster headache: study protocol
}

Jin Li, ${ }^{1}$ Hao Ren, ${ }^{1}$ Baoguo Wang, ${ }^{2}$ Dasheng Wu, ${ }^{3}$ Fang Luo ${ }^{4}$

To cite: Li J, Ren H, Wang B, et al. Multicentre, prospective randomised, controlled, blinded-endpoint study to evaluate the efficacy and safety of pterygopalatine ganglion pulsed radiofrequency treatment for cluster headache: study protocol. BMJ Open 2019;9:e026608. doi:10.1136/ bmjopen-2018-026608

- Prepublication history for this paper is available online To view these files, please visit the journal online (http://dx.doi org/10.1136/bmjopen-2018026608).

$\mathrm{JL}$ and HR contributed equally.

Received 11 September 2018 Revised 21 January 2019 Accepted 13 February 2019

Check for updates

(C) Author(s) (or their employer(s)) 2019. Re-use permitted under CC BY-NC. No commercial re-use. See rights and permissions. Published by BMJ.

For numbered affiliations see end of article.

Correspondence to

Professor Fang Luo;

luofangwt@yahoo.com

\section{ABSTRACT}

Introduction Single-centre reports on small groups of patients have shown that pterygopalatine ganglion pulsed radiofrequency treatment in patients with refractory cluster headache $(\mathrm{CH})$ can quickly relieve pain without significant side effects. However, a randomised controlled trial is still necessary to evaluate whether pterygopalatine ganglion pulsed radiofrequency (PRF) treatment is a viable treatment option for patients with $\mathrm{CH}$ who are not responding to drug treatment.

Methods and analysis This investigation is a multicentre, prospective, randomised, controlled, blinded-endpoint study. We will enrol 80 patients with $\mathrm{CH}$ who are not responding to medication. The enrolled patients will be randomly divided into two groups: the nerve block (NB) group and the PRF group. All patients will undergo CTguided pterygopalatine ganglion puncture. A mixture containing steroids and local anaesthetics will be slowly injected into the patients in the NB group. The patients in the PRF group will be treated with PRF at $42^{\circ} \mathrm{C}$ for $360 \mathrm{~s}$. After treatment, the duration of cluster periods; degree of pain during headache attacks; frequency of headache attacks; duration of each headache attack; dose of auxiliary analgesic drugs; duration of remission; degree of patient satisfaction; effectiveness rates at 1 day, 3 days, 1 week, 2 weeks, 1 month, 3 months, 6 months, and 1 year after the procedure; and intraoperative and postoperative adverse events will be compared between the two groups. Ethics and dissemination This study was approved by the institutional ethics committee of the Beijing Tiantan Hospital (approval number: KY 2018-027-02). The results of the study will be published in peer-reviewed journals, and the findings will be presented at scientific meetings. Trial registration number NCT03567590; Pre-results.

\section{INTRODUCTION}

Cluster headache $(\mathrm{CH})$, a primary headache characterised by severe pain, has a considerable impact on quality of life. $\mathrm{CH}$ attacks are very painful, and the patients can even become suicidal. Therefore, $\mathrm{CH}$ is
Strengths and limitations of this study

This multicentre, prospective, randomised, controlled, blinded-endpoint study will be the first investigation to compare the efficacy of pulsed radiofrequency to that of nerve block with local anaesthetic plus corticosteroids for patients with cluster headache.

- The randomised controlled trial design minimises the risk of confounding bias.

- The participants in this study and the doctors who conduct the interventions will not be blinded to the treatment procedure.

- The follow-up will be performed by telephone instead of hospital visits; telephone follow-up is sufficient to assess the primary outcome.

also known as 'suicidal headache'. ${ }^{2}$ Severe unilateral pain occurs in the orbital, frontal and temporal areas during $\mathrm{CH}$ attacks and can last 15 min to 3 hours, accompanied by ipsilateral oculofacial autonomic symptoms. $\mathrm{CH}$ includes two categories: episodic cluster headache (ECH) and chronic cluster headache $(\mathrm{CCH})$. ECH can involve several attacks in a day, and an attack period of 2 weeks to 3 months is called a 'cluster period', which is followed by a pain-free 'remission period' of $\geq 3$ months. ${ }^{3}$ During the cluster period, headaches often recur every day at a fixed time. Generally, CCH attacks occur less frequently than ECH attacks on a daily basis and have no remission period.

Since the pathogenesis of $\mathrm{CH}$ remains unclear, there is a dearth of targeted aetiological treatment. ${ }^{4}$ It is currently believed that the pathogenesis of $\mathrm{CH}$ may involve the trigeminovascular system and the activation of the parasympathetic system and 
ipsilateral hypothalamic grey matter. ${ }^{45}$ The pterygopalatine ganglion, also known as the sphenopalatine ganglion or ganglion pterygopalatinum, plays a very important role in the pathophysiology of $\mathrm{CH}^{6}{ }^{6}$

The clinical treatment of $\mathrm{CH}$ is still extremely difficult. For patients who do not respond to drug therapy, a pterygopalatine ganglion block via the application of local anaesthetics and steroid hormones has a certain degree of effectiveness. ${ }^{7}$ Generally, a single pterygopalatine ganglion block is not sufficient to achieve satisfactory results; therefore, several such blocks are required, which increases the risk of puncture and steroid-hormone-related side effects. In addition, multiple punctures also increase medical costs. For intractable $\mathrm{CH}$ that does not respond to conservative treatment, deep brain stimulation, ${ }^{8}$ pterygopalatine ganglion ablation ${ }^{9}$ and pterygopalatine ganglion electrical stimulation ${ }^{10-13}$ can provide relief in some patients. Research has shown that tonic stimulation of the pterygopalatine ganglion has preventive effects. ${ }^{10} 13$ However, the abovementioned methods all have problems, such as trauma caused by surgery, serious side effects and high medical costs. Therefore, there is an urgent need for exploring new minimally invasive, safe and effective technologies for the treatment of $\mathrm{CH}$ in clinical practice.

The technique of percutaneous pulsed radiofrequency (PRF) is a minimally destructive pain-treatment technology. ${ }^{14}$ In contrast to radiofrequency thermocoagulation, PRF uses the following parameters: pulse frequency of $2 \mathrm{~Hz}$, output voltage of $45 \mathrm{~V}$, output frequency of $500 \mathrm{kHz}$, continuous current action of $20 \mathrm{~ms}$, intermittent time of $480 \mathrm{~ms}$ and treatment temperature not exceeding $42^{\circ} \mathrm{C}$. This intervention technology does not cause local tissue damage, and there are few side effects.

In 2011, Chua et al first reported the use of pterygopalatine ganglion PRF treatment in three patients with $\mathrm{CH}$, of whom two patients had complete remission of pain, one patient had partial remission of pain, and all patients were free of neurological side effects and complications after treatment. ${ }^{15}$ In 2016, we reported CT-guided pterygopalatine ganglion PRF treatment in 16 patients with $\mathrm{CH}$ who had not responded to drugs and nerve block (NB); we found that pterygopalatine ganglion PRF could quickly, safely and effectively relieve the patients from their $\mathrm{CH}$ periods. ${ }^{16}$ However, to verify whether early intervention via PRF is a viable treatment option for patients with $\mathrm{CH}$ who are not responding to drug therapy, we still need to obtain strong evidence through a properly designed randomised controlled trial. Therefore, this study proposes a multicentre, prospective, randomised, controlled, blinded-endpoint study to compare the pain-relief effects of CT-guided $\mathrm{PRF}$ and NB of the pterygopalatine ganglion for patients with $\mathrm{CH}$ who are not responding to drug treatment. Study outcomes at different timepoints will be assessed with standardised forms and procedures by responsible physicians blinded to the treatment allocation (blinded endpoint).

\section{METHODS}

\section{Trial design}

This investigation is a multicentre, prospective, randomised, controlled, blinded-endpoint study. Patients with $\mathrm{CH}$ who are not responding to drug therapy will receive either CT-guided percutaneous puncture pterygopalatine ganglion NB or PRF, and efficacy and safety will be compared between the two groups of patients (figure 1).

\section{Setting}

Patients will be selected from three research centres: Beijing Tiantan Hospital, Beijing Sanbo Brain Hospital and Jilin Province People's Hospital. All researchers will be trained based on the same training protocol and required to have more than 1 year of clinical experience with each treatment method prior to participating in the study. This clinical study follows the relevant regulations of the Declaration of Helsinki (version 19 October 2013) of the World Medical Association. All patients will sign an informed consent at a screening visit. This study, which has begun on 5 July 2018, will last for 3 years.

\section{Participants}

Suitable participants will be screened at the pain management centre of each hospital to participate in the study.

The inclusion criteria comprise the following: (1) the diagnosis of $\mathrm{CH}$ is confirmed according to the diagnostic criteria of the International Classification of Headache Disorders third edition ${ }^{3}$ (box 1); (2) the patient's age is between 18 and 60 years; (3) the patient seeks treatment in the pain clinics of hospitals participating in the study within 5 days of the onset of the cluster period; the patient's pain condition remains the same after preventive therapy with drugs available in our hospital such as verapamil, topiramate, lithium or steroids, or there is a reduction of less than $50 \%$ in the intensity and frequency of headache attacks, the duration of each attack and the dosage of auxiliary analgesic drugs used.

The exclusion criteria include the following: (1) abnormalities in blood measurements, liver and kidney function, blood glucose, coagulation, electrocardiography or chest radiography; (2) infection at the puncture site; (3) previous mental illness; (4) previous history of narcotic drug abuse; (5) prior anticoagulant or antiplatelet therapy; (6) an implantable pulse generator; (7) previous history of invasive treatments such as pterygopalatine ganglion radiofrequency thermocoagulation and chemical destruction and (8) current pregnancy or breast feeding.

\section{Recruitment and informed consent}

All enrolled patients will have the right to be informed of the purpose of the study, the experimental procedures, the benefits to the participants and the possible risks, after which they will sign the informed consent. All patients will be given enough time to consider whether they would like to participate in this study. Patients participating in the study will also have the right to freely obtain more information at any time and will be allowed to freely 


\section{Enrolment}

Allocated to pulsed radiofrequency treatment group $(n=40)$

Follow-Up

\section{Analysis}

Figure 1 Flow diagram of the study.

withdraw their consent form or withdraw from the study without restrictions at any stage.

Once each patient signs the informed consent, the researchers will complete the eligibility checklists based on the items listed on the case report form; records will be made of any candidates who fail to enrol.

\section{Interventions}

Randomisation and allocation concealment

All participants will be randomly divided into two groups in a 1:1 ratio. The researchers will apply randomisation at each of the three centres. After each enrolled patient is confirmed to satisfy the baseline inclusion and exclusion criteria, the patient will be randomly assigned to one of the two study groups. The random sequence will be generated using SAS V.9.1.3 (SAS Institute) software.

Each research centre will have a research nurse responsible for implementing the allocation. According to a pregenerated random sequence, each enrolled patient will be given a sealed opaque envelope based on the order of enrolment. After the pterygopalatine ganglion is punctured during the procedure, the research nurse will open the sealed envelope and assign the patient to the
Patients with cluster headache that are not responding to mediations $(n=80)$

Randomised

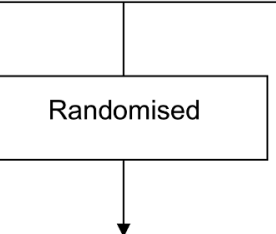

S

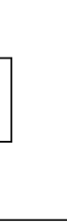

Primary outcome:

econdary outcomes:

- The degree of pain during headache attacks

Duration of remission period

- Effective rates

Adverse events

Statistical analysis, writing and publishing corresponding group according to the random number in the envelope, and the corresponding treatment will then be performed on the patient.

\section{Blinding}

This study has an open-label design. In this investigation, participants and doctors could not all be blinded to the study conditions. However, the telephone follow-ups at different time points after the procedure will be conducted by responsible physicians blinded to the allocation status of the patients. The data input will be completed by dataentry personnel who are not on the research team, and the data analysis will be completed by statisticians blinded to the allocation information.

\section{Study interventions}

The patient will be in a supine position on a CT scanner couch with the head turned approximately $50^{\circ}$ to the contralateral side. Blood pressure, heart rate, electrocardiography and pulse oximetry will be continuously monitored. The negative plate of the PMG-230 pain-treatment generator (Baylis Medical, Montreal, Canada) will be applied to the upper abdominal skin of the patient. 
Box 1 Diagnostic criteria for cluster headache in the International Classification of Headache Disorders third edition (ICHD-3) ${ }^{3}$

\section{Cluster headache}

A. At least five attacks fulfilling criteria B-D

B. Severe or very severe unilateral orbital, supraorbital and/or temporal pain lasting $15-180 \mathrm{~min}$ (when untreated)

C. Either or both of the following:

1. at least one of the following symptoms or signs, ipsilateral to the headache a. conjunctival injection and/or lacrimation; b. nasal congestion and/or rhinorrhoea; c. eyelid oedema; d. forehead and facial sweating; e. miosis and/or ptosis

2. a sense of restlessness or agitation

D. Occurring with a frequency between one every other day and eight per day

E. Not better accounted for by another ICHD-3 diagnosis

\section{Episodic cluster headache}

A. Attacks fulfilling criteria for cluster headache and occurring in bouts (cluster periods)

B. At least two cluster periods lasting from 7 days to 1 year (when untreated) and separated by pain-free remission periods of $\geq 3$ months

\section{Chronic cluster headache}

A. Attacks fulfilling criteria for cluster headache and criterion B below

B. Occurring without a remission period, or with remissions lasting $<3$ months, for at least 1 year

Aseptic drapes will be applied to the patient's face in a routine manner. The puncture point will be located under the zygomatic arch of the affected side, $3-4 \mathrm{~cm}$ in front of the tragus. After the administration of $1 \%$ lidocaine for local anaesthesia, a 21-gauge trocar needle with a length of $10 \mathrm{~cm}$ will be inserted vertically into the puncture point. At an approximate depth of $4 \mathrm{~cm}$, the trocar needle will reach the bone surface of the lateral pterygoid plate of the sphenoid. The trocar needle will be then withdrawn by $2 \mathrm{~cm}$ and reinserted towards the upper part of the middle third of the pterygopalatine fossa until the tip of the needle glides over the leading edge of the lateral pterygoid plate of the sphenoid. The needle will then be inserted another $0.5 \mathrm{~cm}$ to enter the pterygopalatine fossa. We will use a CT scanner (medical X-ray CT scanner, model Somatom, Siemens, Munich, Germany) during the procedure to verify the position of the puncture needle in the pterygopalatine fossa. The orientation and depth of the puncture needle will be adjusted according to the CT image until the needle approaches the pterygopalatine ganglion. The stylet will be removed, and the electrode needle for PRF treatment (PMF-21100-5, Baylis Medical) will be placed. The pain-treatment generator will be connected to the radiofrequency needle, and the sensory threshold will be measured with $50 \mathrm{~Hz}$ electrical stimulation. Induction of sensory abnormality at the root of the nose by a stimulus of $0.1-0.3 \mathrm{~V}$ will indicate accurate puncture, and the depth and direction of the puncture needle will be appropriately adjusted according to the patient's response. When the needle is in place, the patients will receive PRF or NB treatment according to the random number in the envelope. The treatments will be as follows.

\section{PRF treatment}

The pulse treatment generator will be set to the automatic pulsed radiofrequency mode, with a temperature of $42^{\circ} \mathrm{C}$, pulse frequency of $2 \mathrm{~Hz}$, pulse width of $20 \mathrm{~ms}$ and treatment duration of $360 \mathrm{~s} .{ }^{16}$

\section{NB treatment}

A mixture of $40 \mathrm{mg}$ of triamcinolone $+2 \mathrm{~mL}$ of $1 \%$ bupivacaine $+2 \mathrm{~mL}$ of $2 \%$ mepivacaine $+1: 100000$ epinephrine will be injected for NB treatment using a puncture needle. ${ }^{1718}$

After the operation, the patients will be delivered to the outpatient recovery room, and they will be discharged if no adverse signs are noted within 2 hours. Verapamil, topiramate, lithium or steroid administration will be discontinued if patients take these medications prior to the procedure. The doctor will use rizatriptan to abort individual attacks as needed. Participants will be treated with salvage therapy using other, more invasive therapies such as pterygopalatine ganglion ablation, electrical stimulation of the pterygopalatine ganglion and deep brain stimulation if the pain and the necessary dosage of auxiliary drugs do not differ from their preoperative levels.

\section{Patient and public involvement}

Neither the patients nor members of the public were involved in the development of the research question, design or outcome measures of this study. The study recruitment will be conducted through research posters and physicians' presentations. Participant screening and enrolment will be performed by trained physicians. The trial outcomes of this study will be disseminated to all participants in a newsletter on request. The burden of the intervention will not be assessed by patients themselves. All participants will be informed in detail of the cost of the relevant intervention.

\section{Variables and measurements}

Prior to the intervention, the age, gender, headache lateralisation (left or right), previous duration of cluster periods, current numeric rating scale (NRS, from 0 points for no pain to 10 points for the most severe pain) score during headache attacks, frequency of headache attacks, duration of each headache attack, dose of auxiliary analgesics and previous duration of remission of the enrolled patients will be recorded, and a prospective evaluation will be conducted by having the patients keep diaries.

The patients will be followed up by telephone at 1 day, 3 days, 1 week, 2 weeks, 1 month, 3 months, 6 months, and 1 year after the procedure by responsible physicians who are blinded to the allocation status of the patients. The primary outcome is the duration of the cluster periods. The duration of a cluster period is defined as the total duration of the headache, including the pain experienced before and after treatment. The secondary 
outcomes, which include the degree of pain during headache attacks (NRS scores), the frequency of headache attacks, the duration of each headache attack, the dose of auxiliary analgesic drugs taken, the duration of remission, self-rated patient satisfaction (from 0 point for unsatisfied to 10 points for very satisfied), the effectiveness rate of treatment and intraoperative and postoperative adverse events (AEs), will also be compared between the two groups of patients.

The effectiveness of the treatments at each time point will be calculated. Effectiveness will be defined as either complete or partial relief of pain, and the rate will be calculated as follows: effectiveness rate $=$ number of effectively treated patients/total number of patients in this group $\times 100 \%$. Complete pain relief will be defined as NRS $=0$ and discontinued administration of drugs. Partial pain relief will be defined as a postoperative reduction of more than $50 \%$ in the intensity, frequency and duration of headache attacks as well as auxiliary analgesic drug dosage. No remission of pain will be defined as no change from the preoperative level of pain or as the postoperative intensity, frequency and duration of headache attacks as well as auxiliary analgesic drug dosage remaining over $50 \%$ of the preoperative levels. The partial pain remission time, complete pain remission time, number of interventional treatments, treatment intervals and number of cases receiving electrical stimulation of the pterygopalatine ganglion will be recorded.

Details regarding the AEs will be recorded during the procedure and at various time points during the postoperative follow-up period ( 1 day, 3 days, 1 week, 2 weeks, 1 month, 3 months, 6 months and 1 year after the procedure). For intraoperative AEs, the occurrence of puncture pain, headache, dizziness, nausea, vomiting, facial haematoma and other effects will be recorded. For postoperative AEs, headache, dizziness, facial numbness and other effects will also be recorded.

\section{Sample size}

This study will apply a one-sided superiority test with $\alpha=0.025$ and $\beta=0.10$. Based on a review of the literature, combined with the authors' published articles and clinical experience, ${ }^{16}$ the duration of the cluster period of the PRF group is approximately 15.5 days, and the SD is 9.3 days, while the cluster duration of the NB group is approximately 45 days, with a SD of approximately 15 days. Shortening the duration of the cluster period by 20 days has clinical significance. The number of cases needed in each group is 36 as calculated by Power Analysis and Sample Size software program (PASS) V.11. Allowing for a $10 \%$ rate of loss to follow-up, 40 cases are required in each group, and a total of 80 cases are required for both groups together.

\section{Statistical analysis}

The statistical analysis software SAS V.9.4 will be used to analyse both the full data set and the per-protocol set. The Shapiro-Wilk test will be used to test whether the data follow the normal distribution. Normally distributed data will be expressed as the means \pm SD deviations. Parameters that do not follow the normal distribution will be expressed as medians \pm quartiles. Student's t-test will be used for measurement data with normal distributions, the Wilcoxon rank sum test will be used for measurement data with non-normal distributions and the $\chi^{2}$ test will be used for count data. Effectiveness will be analysed via both intention-to-treat analysis and the per-protocol analysis set in SAS. Student's t-test will be used to compare measurement data on the outcome indicators, such as duration of cluster periods, degree of during headache attacks, frequency of headache attacks, duration per headache attack, dose of auxiliary analgesics, duration of remission and patient satisfaction, between the PRF group and the NB group. The $\chi^{2}$ test will be used to compare the count data of efficacy outcome indicators between the PRF and NB groups. The $\chi^{2}$ test or Fisher's exact test will be used to evaluate intraoperative and postoperative AEs.

\section{DISCUSSION}

The pterygopalatine ganglion is one of the four major parasympathetic ganglia of the head and neck. This ganglion is the largest group of neurons within the calvarium outside the brain and is the only ganglion that enters the external environment through the nasal mucosa. ${ }^{19}$ The characteristic clinical symptoms of $\mathrm{CH}$, such as tearing, runny nose, nasal congestion and nasal oedema, are manifestations of parasympathetic excitation in the pterygopalatine ganglion, and ptosis and pupil diminution are manifestations of sympathetic inhibition in the pterygopalatine ganglion. Therefore, the pathogenesis of $\mathrm{CH}$ is considered to be related to the pterygopalatine ganglion. ${ }^{20}$

In recent years, there have been a series of reports on the treatment of $\mathrm{CH}$ via the pterygopalatine ganglion. One type of pterygopalatine ganglion treatment is destructive treatment, which blocks pain signalling by denaturing pterygopalatine ganglion proteins; treatments of this type include radiofrequency ablation techniques ${ }^{21} 22$ and local injection of absolute alcohol. ${ }^{2324}$ The other type is minimally destructive treatment, such as $\mathrm{NB},{ }^{10}{ }^{20} \mathrm{PRF}^{16}$ and electrical nerve stimulation. ${ }^{1325}$

Methods of pterygopalatine ganglion NB include cottonswab nasal infiltration ${ }^{26}$ and needle injection. ${ }^{17}{ }^{18}$ Puncture approaches to the pterygopalatine ganglion include the sphenopalatine foramen approach, ${ }^{27}$ the suprazygomatic approach, ${ }^{23}$ the infrazygomatic crest approach ${ }^{24}$ and the mandibular notch approach. ${ }^{28}$ Puncture can be performed with the assistance of nasal endoscopy, ${ }^{17} 18$ fluoroscopy ${ }^{21} 22$ or CT. ${ }^{916}$ The commonly used NB drugs include local anaesthetics and steroid hormones. The previous reports on $\mathrm{NB}$ for the treatment of $\mathrm{CH}$ are mostly case-series analyses and case reports. Costa et al conducted a randomised double-blind placebo-controlled study of patients with nitroglycerine-induced $\mathrm{CH}$; the patients were treated with $10 \%$ cocaine, $10 \%$ lidocaine 
or a saline placebo. ${ }^{26}$ The results showed that short-term treatment effects were significant in the cocaine and lidocaine groups, and there were no related acute side effects. However, NB treatment for $\mathrm{CH}$ has certain drawbacks, the most important of which are the limited effect of pain relief in a single treatment and the short duration of treatment efficacy. Therefore, NB needs to be implemented repeatedly. The puncture approach in our study will be the infrazygomatic crest approach, ${ }^{24}$ also known as the translateral approach, and the injection will be a mixture of the steroid hormone triamcinolone acetonide and local anaesthetics in the control group.

PRF is a minimally destructive, minimally invasive, percutaneous interventional pain management technique. ${ }^{29}$ This procedure uses the same puncture site and localisation approach as radiofrequency ablation. However, to treat the pain, PRF regulates the nerve function through an electric field, while radiofrequency ablation destroys the nerve by thermal damage. Our previous study found that after PRF treatment of 16 patients with $\mathrm{CH}$ who had not responded to drugs or NB, 11 patients with $\mathrm{ECH}$ and 1 patient with $\mathrm{CCH}$ had complete remission, although treatment was ineffective for 2 patients with $\mathrm{ECH}$ and 2 patients with $\mathrm{CCH} .{ }^{16}$ Bendersky et al also reported that PRF treatment failed to achieve satisfactory pain relief in three patients with $\mathrm{CCH} .{ }^{30}$ Therefore, it is currently believed that PRF treatment may be more effective for ECH than for CCH. However, given the low incidence of $\mathrm{CCH}$ and the small number of established cases, the existing studies are not sufficient to reach a convincing conclusion.

CT images are clear and intuitive, providing the clinician with accurate guidance for puncturing the surgical site. The CT-guided pterygopalatine ganglion puncture technique was first applied in clinical practice by Kastler et $a l^{24}$ CT guidance was confirmed to reduce puncture complications and increase both the puncture success rate and treatment satisfaction. In the past, we have reported a $100 \%$ success rate of puncture for patients with $\mathrm{CH}$ undergoing CT-guided pterygopalatine ganglion puncture and PRF treatment, and surgery-related complications, such as nosebleeds and cheek haematomas, were successfully avoided ${ }^{16}$ In the proposed study, both the NB and PRF groups will undergo CT-guided pterygopalatine ganglion puncture to ensure the accuracy of the puncture and to avoid the effects of inaccurate puncture on the outcome. During the procedure, the dose of radiation exposure will be controlled by minimising the scope of CT scans, for example, scanning only the pterygopalatine fossa as needed by the experienced physician performing the puncture.

This study will compare the effectiveness of pterygopalatine ganglion NB and PRF for the treatment of $\mathrm{CH}$ in a multicentre, prospective, randomised, controlled, blinded-endpoint study; the results are expected to provide reliable evidence regarding treatment strategies for patients with $\mathrm{CH}$ who are not responding to conservative drug treatment. Of course, this study has some limitations; for example, the participants in the trial and the doctors who conduct the interventions will be not kept blinded to the treatment allocations. Double-blind studies need to be carried out in the future to achieve results of greater scientific value. Other limitations include the short follow-up period of only 1 year and the lack of exploration of optimal parameters for PRF treatment of $\mathrm{CH}$, which will be investigated through in-depth clinical research later. Furthermore, the variability in the duration of episodes in patients with $\mathrm{CH}$ makes it difficult to discern the response pattern.

\section{Author affiliations}

${ }^{1}$ Department of Anesthesiology and Pain Management, Beijing Tiantan Hospital, Capital Medical University, Beijing, China

${ }^{2}$ Department of Anesthesiology, Beijing Sanbo Brian Hospital, Capital Medical University, Beijing, China

${ }^{3}$ Department of Pain Management, Jilin Province People's Hospital, Changchun, China

${ }^{4}$ Department of Pain Management, Beijing Tiantan Hospital, Capital Medical University, Beijing, China

Contributors $\mathrm{JL}$ and HR contributed equally to this work and should be considered co-first authors. They wrote the protocol and this manuscript. FL is the principal investigator of the whole study. FL, BW and DW are the site principal investigator of each research centre. FL, JL and HR contributed to the conception and design of the research protocol. All authors approved the final version to be published.

Funding This work was supported by the Beijing Municipal Administration of Hospitals Clinical Medicine Development of Special Funding Support (grant no. XMLX201707) and the Foundation for the Excellent Medical Staff of Beijing (grant no. 2014-3-035).

Competing interests None declared.

Patient consent for publication Not required.

Provenance and peer review Not commissioned; externally peer reviewed.

Open access This is an open access article distributed in accordance with the Creative Commons Attribution Non Commercial (CC BY-NC 4.0) license, which permits others to distribute, remix, adapt, build upon this work non-commercially, and license their derivative works on different terms, provided the original work is properly cited, appropriate credit is given, any changes made indicated, and the use is non-commercial. See: http://creativecommons.org/licenses/by-nc/4.0/.

\section{REFERENCES}

1. Rozen TD, Fishman RS. Cluster headache in the United States of America: demographics, clinical characteristics, triggers, suicidality, and personal burden. Headache 2012;52:99-113.

2. Weaver-Agostoni J. Cluster headache. Am Fam Physician 2013;88:122-8.

3. (IHS) HCCotlHS. Headache Classification Committee of the International Headache Society (IHS) The International Classification of Headache Disorders, 3rd edition. Cephalalgia 2018;38:1-211

4. Hoffmann J, May A. Diagnosis, pathophysiology, and management of cluster headache. Lancet Neurol 2018;17:75-83.

5. May A, Bahra A, Büchel C, et al. Hypothalamic activation in cluster headache attacks. Lancet 1998;352:275-8.

6. Schytz HW, Barløse M, Guo S, et al. Experimental activation of the sphenopalatine ganglion provokes cluster-like attacks in humans. Cephalalgia 2013;33:831-41.

7. Peñarrocha-Diago M, Boronat A, Peñarrocha-Oltra D, et al. Clinical course of patients with episodic cluster headache treated with corticosteroids inproximity to the sphenopalatine ganglion: a preliminary study of 23 patients. Med Oral Patol Oral Cir Bucal 2012;17:e477-82.

8. Lepper A, Frese A, Summ O, et al. Hypothalamic dopaminergic stimulation in cluster headache. Cephalalgia 2013;33:1155-9. 
9. Loomba V, Upadhyay A, Kaveeshvar H. Radiofrequency ablation of the sphenopalatine ganglion using cone beam computed tomography for intractable cluster headache. Pain Physician 2016;19:E1093-6.

10. Barloese MC, Jürgens TP, May A, et al. Cluster headache attack remission with sphenopalatine ganglion stimulation: experiences in chronic cluster headache patients through 24 months. J Headache Pain 2016;17:67.

11. Kohlmeier $\mathrm{C}$, Behrens $\mathrm{P}$, Böger $\mathrm{A}$, et al. Improved surgical procedure using intraoperative navigation for the implantation of the SPG microstimulator in patients with chronic cluster headache. Int $J$ Comput Assist Radiol Surg 2017;12:2119-28.

12. Meng DW, Zhang JG, Zheng Z, et al. Chronic bilateral sphenopalatine ganglion stimulation for intractable bilateral chronic cluster headache: a case report. Pain Physician 2016;19:E637-42.

13. Schoenen J, Jensen RH, Lantéri-Minet $M$, et al. Stimulation of the sphenopalatine ganglion (SPG) for cluster headache treatment. Pathway $\mathrm{CH}-1$ : a randomized, sham-controlled study. Cephalalgia 2013;33:816-30

14. Sluijter ME. Non-thermal radiofrequency procedures in the treatment of spinal pain. Pain in Europe. Barcelona: 2nd Annual Congress of the European Federation of IASP Chapters, 1997:326.

15. Chua NH, Vissers KC, Wilder-Smith $\mathrm{OH}$. Quantitative sensory testing may predict response to sphenopalatine ganglion pulsed radiofrequency treatment in cluster headaches: a case series. Pain Pract 2011;11:439-45.

16. Fang L, Jingjing L, Ying S, et al. Computerized tomography-guided sphenopalatine ganglion pulsed radiofrequency treatment in 16 patients with refractory cluster headaches: Twelve- to 30-month follow-up evaluations. Cephalalgia 2016;36:106-12.

17. Felisati G, Arnone F, Lozza P, et al. Sphenopalatine endoscopic ganglion block: a revision of a traditional technique for cluster headache. Laryngoscope 2006;116:1447-50.

18. Pipolo C, Bussone G, Leone M, et al. Sphenopalatine endoscopic ganglion block in cluster headache: a reevaluation of the procedure after 5 years. Neurol Sci 2010;31:197-9.
19. Ho KWD, Przkora R, Kumar S. Sphenopalatine ganglion: block, radiofrequency ablation and neurostimulation - a systematic review. $J$ Headache Pain 2017;18:118.

20. Piagkou M, Demesticha T, Troupis T, et al. The pterygopalatine ganglion and its role in various pain syndromes: from anatomy to clinical practice. Pain Pract 2012;12:399-412.

21. Narouze S, Kapural L, Casanova J, et al. Sphenopalatine ganglion radiofrequency ablation for the management of chronic cluster headache. Headache 2009:49:571-7.

22. Oomen KP, van Wijck AJ, Hordijk GJ, et al. Effects of radiofrequency thermocoagulation of the sphenopalatine ganglion on headache and facial pain: correlation with diagnosis. $J$ Orofac Pain 2012;26:59-64.

23. Devoghel JC. Cluster headache and sphenopalatine block. Acta Anaesthesiol Belg 1981;32:101-7.

24. Kastler A, Cadel G, Comte A, et al. Alcohol percutaneous neurolysis of the sphenopalatine ganglion in the management of refractory cranio-facial pain. Neuroradiology 2014;56:589-96.

25. Jürgens TP, Barloese M, May A, et al. Long-term effectiveness of sphenopalatine ganglion stimulation for cluster headache. Cephalalgia 2017;37:423-34.

26. Costa A, Pucci E, Antonaci F, et al. The effect of intranasal cocaine and lidocaine on nitroglycerin-induced attacks in cluster headache. Cephalalgia 2000;20:85-91.

27. Barré F. Cocaine as an abortive agent in cluster headache. Headache 1982;22:69-73.

28. Zarembinski C, Graff-Radford S. An unusual challenge in performing sphenopalatine ganglion block with enlarged coronoid process: Jacob's disease. Pain Med 2014;15:329-32.

29. Vanneste T, Van Lantschoot A, Van Boxem K, et al. Pulsed radiofrequency in chronic pain. Curr Opin Anaesthesiol 2017;30:577-82.

30. Bendersky DC, Hem SM, Yampolsky CG. Unsuccessful pulsed radiofrequency of the sphenopalatine ganglion in patients with chronic cluster headache and subsequent successful thermocoagulation. Pain Pract 2015;15:E40-5. 